\title{
Huntington's Disease Protein Huntingtin Associates with its own mRNA
}

\author{
Brady P. Culver a,1, Josh DeClercq ${ }^{\mathrm{a}}$, Igor Dolgalev ${ }^{\mathrm{c}}$, Man Shan $\mathrm{Yu}^{\mathrm{a}}$, Bin Ma ${ }^{\mathrm{a}, 2}$, \\ Adriana Heguy ${ }^{\mathrm{b}, \mathrm{c}}$ and Naoko Tanese ${ }^{\mathrm{a}, *}$ \\ ${ }^{a}$ Department of Microbiology, New York University School of Medicine, New York, NY, USA \\ ${ }^{\mathrm{b}}$ Department of Pathology, New York University School of Medicine, New York, NY, USA \\ ${ }^{\mathrm{c}}$ Genome Technology Center, New York University School of Medicine, New York, NY, USA
}

\begin{abstract}
.
Background: The Huntington's disease (HD) protein huntingtin (Htt) plays a role in multiple cellular pathways. Deregulation of one or more of these pathways by the mutant Htt protein has been suggested to contribute to the disease pathogenesis. Our recent discovery-based proteomics studies have uncovered RNA binding proteins and translation factors associated with the endogenous Htt protein purified from mouse brains, suggesting a potential new role for Htt in RNA transport and translation. Objective: To investigate how Htt might affect RNA metabolism we set out to purify and analyze RNA associated with Htt. Methods: RNA was extracted from immunopurified Htt-containing protein complexes and analyzed by microarrays and RNA-Seq.

Results: Surprisingly, the most enriched mRNA that co-purified with Htt was Htt mRNA itself. The association of Htt protein and Htt mRNA was detected independent of intact ribosomes suggesting that it is not an RNA undergoing translation. Furthermore, we identified the recently reported mis-spliced Htt mRNA encoding a truncated protein comprised of exon 1 and a portion of the downstream intron in the immunoprecipitates containing mutant Htt protein. We show that Htt protein co-localizes with $H t t$ mRNA and that wild-type Htt reduces expression of a reporter construct harboring the Htt 3' UTR.

Conclusions: HD protein is found in a complex with its own mRNA and RNA binding proteins and translation factors. Htt may be involved in modulating its expression through post-transcriptional pathways. It is possible that Htt shares mechanistic properties similar to RNA binding proteins such as TDP-43 and FUS implicated in other neurodegenerative diseases.
\end{abstract}

Keywords: Huntington's disease, huntingtin exon 1, RNA-protein interaction, mRNA translation, Fmrp

\section{INTRODUCTION}

Huntington's disease (HD) is an inherited neurodegenerative disorder caused by a CAG repeat

\footnotetext{
${ }^{1}$ Current position: Enzymatics, Boulder, CO 80303

${ }^{2}$ Current position: School of Veterinary and Life Sciences, Murdoch University, Murdoch, Australia

*Correspondence to: Naoko Tanese, Department of Microbiology, New York University School of Medicine, 550 First Ave., New York, NY 10016, USA. Tel.: +1 212263 8945; Fax: +1 212 263 8276; E-mail: tanesn01@med.nyu.edu.
}

expansion in the coding region of the Huntington's disease gene huntingtin (HTT), which results in an increased number of glutamine residues within the $\mathrm{N}$-terminus of the huntingtin protein $\mathrm{Htt}$ [1]. The onset of HD symptoms is temporally related to the degree of CAG repeat expansion; longer repeat expansions usually correlate with an earlier appearance of symptoms [2]. HD typically manifests during mid-life and is characterized by chorea, impaired mental abilities, altered personality, and is ultimately fatal. Post-mortem examination of the brains of 
affected individuals shows massive cell loss in caudate, putamen, and to a lesser extent the cortex, indicating that these neurons are particularly sensitive to the toxic effects of mutant $\mathrm{Htt}$, despite the near ubiquitous expression of the mutant allele [3]. Currently there is no cure for HD as we have an incomplete understanding of the mechanisms of the disease. We must therefore better understand $\mathrm{Htt}$ function if effective therapies or cures are to be developed.

Investigations into the function of wild type and mutant $\mathrm{Htt}$ have identified roles for the proteins in transcription, axonal transport, cytoskeletal structure/ function, signal transduction, autophagy, and recently post-transcriptional gene expression [4-7]. Htt involvement in gene expression could contribute to the specific pattern of cell loss and symptoms seen in HD if particular groups of genes/messages are more adversely affected over others. These potential changes in gene expression patterns need not be large in magnitude; they are likely small to account for the delay in symptom appearance, and by all accounts apparently normal development is observed in affected individuals. Our recent works have implicated $\mathrm{Htt}$ in Processing (P)-body function, RNA transport, and protein synthesis. Htt associates with Ago2 and contributes to post-transcriptional gene silencing [7]. We find mRNA transport inhibited in cultured rat neurons upon knockdown of $\mathrm{Htt}$ [6], and $\mathrm{Htt}$ co-localizes with mRNAs for $\beta$-actin, BDNF, and microtubule-dependent motor proteins Kif5a and Dhc [8,9]. Furthermore, wild type and mutant $\mathrm{Htt}$ co-purify with large numbers of RNA binding proteins in cytoplasmic mouse brain purifications [4]. These observations support the possibility that $\mathrm{Htt}$ influences gene expression at steps downstream of transcription.

Given the preponderance of evidence implicating Htt in RNA metabolism, we sought to identify mRNAs that specifically associate with $\mathrm{Htt}$ in the mouse brain using 3xFLAG tagged Htt knock-in mice [4]. We reasoned that differences in RNA associations could partially contribute to the tissue specific effects of HD. In our initial microarray analysis of co-purifying mRNAs from FLAG-Htt affinity purifications, we repeatedly identified the $H t t$ mRNA as a co-purifying message. The results were also confirmed by RNA-seq. Here we show that Htt proteins co-localize with $\mathrm{Htt}$ mRNA and that wild-type $\mathrm{Htt}$ confers down regulation of a reporter gene containing the Htt 3' UTR sequence.

\section{MATERIALS AND METHODS}

\section{Htt affinity purifications and RNA isolation}

FLAG-Htt affinity purifications were conducted essentially as described [4] with a few modifications. After the $1400 \times g$ spin, Triton X-100 was added to $\mathrm{S} 1$ lysates to a final concentration of $0.5 \%$. Lysates were incubated at $4{ }^{\circ} \mathrm{C}$ for 20 minutes, and centrifuged at $14,000 \times g$ for 20 minutes to give a total cytoplasmic lysate. EDTA was added to one half of the lysate at a final concentration of $20 \mathrm{mM}$ or an equivalent volume of lysis buffer, and incubated at $4{ }^{\circ} \mathrm{C}$ for 20 minutes. Protein concentration was measured by Bradford assay and adjusted to $6 \mathrm{mg} / \mathrm{mL}$ with lysis buffer prior to binding to $80 \mu \mathrm{L}$ (packed bead volume) of Anti-FLAG M2 agarose beads (Sigma) for 3 hours at $4{ }^{\circ} \mathrm{C}$ with gentle mixing. Beads were washed and eluted as described [4]. Endogenous Htt immunoprecipitations from Neuro-2a cells was performed also as described [4]. Eluted materials from both types of purifications were extracted with $0.5 \mathrm{~mL}$ of TRIReagent (Sigma) and processed to isolate RNA and proteins as per manufacturer's instructions. RNA was further cleaned using a Direct-zol RNA miniprep column (Zymo Research). Eluted RNA was concentrated by ethanol precipitation in the presence of $5 \mu \mathrm{g}$ of linear acrylamide. $20 \%$ of eluted proteins were examined for Htt recovery by western blotting and the remainder used to probe for Htt-associated proteins. Mouse brains were obtained in accordance with the guidelines established by the NIH for the care of laboratory animals, and all procedures approved by Institutional Animal Care and Use Committee (Protocol 140308-01).

\section{cDNA synthesis, qPCR oligonucleotides}

Total cDNA was generated from $1 \mu \mathrm{g}$ of inputs and the entirety of immunoprecipitations using a cDNA synthesis kit and the 10x primer mix from USB (Affymetrix). Equal volumes of cDNA synthesis reactions were used in RT-qPCR reactions using the $2 \mathrm{X}$ SYBR green master mix from USB (Affymetrix). qPCR data was calculated relative to a Gapdh (Htt IP) or Htt (FLAG IP) standard curves from input cDNAs. All qPCR reactions were performed in triplicate and trends were reproduced with experimental replicates. The oligonucleotides used in the PCR reactions were as follows. 
$H t t$ 3' UTR Forward: TAGAGCCATCGAAGGA ACTGCTCCC

Htt 3' UTR Reverse: GCATACAGCAGCAAGGC TTGGACAG

Gapdh Forward: GGTGCTGAGTATGTCGTGGA Gapdh Reverse: CGGAGATGATGACCCTTTTG For $3 \times$ FLAG-Htt ${ }^{7 \mathrm{Q} / 7 \mathrm{Q}} \mathrm{mESCs}$, total RNA and FLAG-IP RNA were extracted with TRI-Reagent. cDNA synthesis and qPCR were carried out as above using the following oligonucleotides.

$\mathrm{Htt}$ exon 1-2 Forward: AGGAACCGCTGCAC CGA

$H t t$ exon 1-2 Reverse: CTGAGAGACTGTGCCA CAATGTT

$H t t$ exon 66-67 Forward: CCAGCCCATGGGTTT CTGCG

$H t t$ exon 66-67 Reverse: AAGCAGCCTGTGGTA TGGACTTCC

Oct4 Forward: GAAGCAGAAGAGGATCACCTT

Oct4 Reverse: TTCTTAAGGCTGAGCTGCAAG

MaoA Forward: GCCCAGTATCACAGGCCAC

MaoA Reverse: CGGGCTTCCAGAACCAAGA

\section{Immunofluorescence microscopy}

Cells were washed $3 x$ in pre-warmed $\left(37^{\circ} \mathrm{C}\right) \mathrm{PBS}$, then fixed in pre-warmed $4 \%$ paraformaldehyde for 10 minutes at $37^{\circ} \mathrm{C}$. Fixed cells were washed $3 \mathrm{x}$ in PBS, then blocked for 1 hour at room temperature in $5 \%$ goat serum, $0.25 \%$ saponin, in PBS. The visualization of Venus did not require the use of antibodies. All incubations in primary antibodies were carried out overnight at $4{ }^{\circ} \mathrm{C}$ and secondary incubations done at room temperature for 1 hour. Coverslips were mounted in Dako fluorescent mounting medium. Images were acquired on a Zeiss META510 confocal scanning laser system on an Axiovert 200M microscope using a Plan-Apochromat 100X/1.3 oilimmersion objective lens. Brightness and contrast settings were adjusted image-wide using Adobe Photoshop.

\section{Antibodies}

For western blotting mouse monoclonal anti-Htt mAB2166 (Millipore) was used at 1:1000, antiRACK1 (Gnb211) (BD Transduction Laboratories) at 1:2500, anti-Rps6 2217 (Cell Signaling Technology) at 1:500, anti-Myo5a (Cell Signaling Technology) 1:1000, anti- $\beta$-tubulin (Covance) $1: 1000$, anti- $\beta$ actin ac15 (Santa Cruz Biotechnology) 1:400, and
anti-GFP (Santa Cruz Biotechnology) at 1:500 in TBST. For immunofluorescence, mouse monoclonal anti-FLAG M2 (Sigma) was used at 1:1000, rabbit polyclonal anti-Htt (Enzo Life Sciences) at 1:1000, and anti-Fmr1 (Millipore) mAB2160 at 1:500. For immunoprecipitations $2 \mu \mathrm{g}$ of anti-Htt (Enzo Life Sciences) was used to immunoprecipitate Htt from Neuro-2a cells or $2 \mu \mathrm{g}$ of rabbit IgG (Santa Cruz Biotechnology) as the negative control.

\section{Plasmids}

The MS2 binding sites of the bacteriophage MS2 protein were cloned downstream of the Renilla luciferase gene in pRL-TK vector (Promega). PCRcloned $H t t$ 3' UTR sequences from a mouse genomic clone containing the entire Htt 3' UTR was inserted downstream of the MS2 binding sites to generate the MS2bs reporter constructs. pNLS-MS2-Venus was obtained from [10] and the FLAG-Ago2 plasmid was obtained from Addgene. Htt 3' UTR sequences were also subcloned downstream of pEGFP-C1 (Clontech) to generate the GFP reporter constructs.

\section{Cell culture and transfections}

Mouse Neuro-2a cells were cultured in $10 \%$ FBS in DMEM (Mediatech) and transiently transfected using Lipofectamine 2000 (Invitrogen) under recommended conditions. Mouse Htt knock-in striatal precursor lines [11] were cultured in $10 \%$ FBS in DMEM and grown at $33^{\circ} \mathrm{C} .3 \mu \mathrm{g}$ of total plasmid DNA/well of a 6-well plate was used in MS2 transfections: $3 \mu \mathrm{g}$ pNLS-Venus-MS2 in control transfections, $800 \mathrm{ng}$ pNLS-MS2-Venus and $2.2 \mu \mathrm{g}$ pRL-MS2bs-Htt-short 3' UTR, and $600 \mathrm{ng}$ pNLS-MS2-Venus and $2.4 \mu \mathrm{g}$ pRL-MS2bs- $H t t$-long 3' UTR. Transfections were performed for 24 hours prior to fixation. For GFP reporter assays, Neuro-2a cells were split into $6 \mathrm{~cm}$ plates. At 24 hours, Htt17Q or CMV-empty control plasmid was transfected at a concentration of $3 \mu \mathrm{g} / \mathrm{plate}$. At 30 hours, cells were split split equally into $4-25 \mathrm{~mm}$ plates. After 48 hours, equimolar amounts of GFP reporters were transfected: $1.18 \mu \mathrm{g}$ GFP-SV40-polyA, $2.1 \mu \mathrm{g}$ GFP$H t t$-long 3' UTR, $1.33 \mu \mathrm{g}$ GFP-Htt-short 3'UTR. Cells were harvested after 72 hours and $20 \mu \mathrm{g}$ of soluble lysates were analyzed by SDS-PAGE followed by immunoblotting with anti-GFP and anti$\mathrm{Htt}$ antibodies and visualized and quantified using Odyssey imager (LI-COR). siRNA transfections 
were performed as described. Transfection of rat cortical neurons and co-localization of endogenous Htt with Htt 3' UTR were performed as previously described $[6,12]$.

\section{Experiments with mouse embryonic stem cell (mESC) lines}

Three lines were used: Wild type, 3xFLAG$\mathrm{Htt}^{7 \mathrm{Q} / 7 \mathrm{Q}}$ and $3 \times \mathrm{FLAG}-\mathrm{Htt}^{140 \mathrm{Q} / 7 \mathrm{Q}}$. mESCs were cultured on gelatin-coated plates in standard ES medium (DMEM supplemented with 15\% ES-Cult FBS, $1 \%$ sodium pyruvate, $1 \%$ NEAA, $1 \%$ penicillinstreptomycin, $0.1 \mathrm{mM}$ 2-mercaptoethanol, and 1000 $\mathrm{U} / \mathrm{ml}$ LIF). Cells were lysed in total lysis buffer [20 mM Tris- $\mathrm{HCl}$ (pH 8.0), $137 \mathrm{mM} \mathrm{NaCl}, 1 \mathrm{mM}$ EDTA, $1 \%$ Triton X-100, $10 \%$ glycerol, $1.5 \mathrm{mM}$ $\mathrm{MgCl}_{2}, 1 \mathrm{mM}$ DTT, $1 \mathrm{mM}$ PMSF, and 1X protease inhibitor cocktail]. Sixty milligrams of total protein was immunoprecipitated with anti-FLAG M2 affinity beads (Sigma A2220) for 4 hours on a nutator at $4^{\circ} \mathrm{C}$. The beads were first washed with total lysis buffer in a column, and then second wash with total lysis buffer w/o Triton X-100. FLAGHtt complexes were eluted with 3X FLAG peptide ( $200 \mathrm{ng} / \mu \mathrm{l}$, Sigma F4799), and concentrated by TCA (trichloroacetic acid) overnight. SDS-PAGE was performed by loading $40 \mu \mathrm{g}$ of total protein for each input sample and 5\% of total eluate for each FLAG-IP sample. Membranes were probed using the following primary antibodies: Mouse anti-FLAGM2 (Sigma F-1804), mouse anti-Htt2166 (Millipore MAB2166) and mouse anti-polyQ (clone 3B5H10, Sigma P1874). IRDye 800CW goat anti-mouse secondary antibody was used (LI-COR Biosciences). Fluorescent signal was scanned using the LI-COR Odyssey machine.

\section{RNA-Seq analysis}

RNA-Seq libraries were made using the SMARTer Ultra Low RNA kit for Illumina (Clontech, Cat \# 634935 and 634936), using 1 to 2 ng of RNA. Libraries were run on an Illumina 2500, using a paired end 50 protocol. Sequencing reads were demultiplexed and converted to FASTQ format using Illumina Bcl2FastQ software. Reads were aligned to the mouse genome (build mm10/GRCm38) using the splice-aware STAR aligner [13]. PCR duplicates were removed using the Picard toolkit (http://broadinstitute.github.io/picard). HTSeq package [14] was utilized to generate counts for each gene and exon based on number of aligned reads overlapping them. These counts were used to test for differential exon usage using negative binomial generalized linear models and for visualization as implemented by the DEXSeq R package [15].

\section{RESULTS}

\section{Purified endogenous Htt complex contains its own $m R N A$}

In previous studies we utilized FLAG-tagged wild type (F7Q/F7Q) and mutant (F140Q/+) Htt knock-in mice [16] to isolate Htt-containing protein complexes from juvenile mouse brains. Co-purifying proteins identified by mass spectrometry revealed many RNA binding proteins and translation factors, suggesting a new role for $\mathrm{Htt}$ in post-transcriptional gene regulatory pathways [4]. The finding prompted us to analyze RNA that might be present in the Htt protein complexes. Surprisingly, microarray and RNA-Seq analyses of co-purifying mRNAs consistently identified the $H t t$ mRNA as the most enriched RNA in our FLAG-Htt purifications from mouse brains compared with the negative control (FLAG-IP eluates of normal mouse brains expressing noFLAG-tagged proteins). Multiple RT-qPCR experiments on co-eluted RNA from FLAG affinity purifications confirmed the drastic enrichment of $H t t$ mRNA in F7Q and F140Q/+ purifications compared with the control. Comparison of the recovery of $\mathrm{Htt}$ mRNA to other mRNAs (Oct 4, $M a o A$, as examples) in the FLAG-IP eluates clearly demonstrated selective retention of the $H t t$ mRNA in the FLAG immunoprecipitates (Supplementary Figure 1). No other mRNAs showed enrichment above the levels found in the control immunoprecipitate, which represented non-specific binding. We wondered whether this interaction might be an artifact of the purification scheme used to purify Htt as the FLAG tag is positioned at the $\mathrm{N}$-terminus of wild type and mutant alleles. It is possible that our Htt purifications recovered $\mathrm{Htt}$ mRNA co-translationally; i.e., incompletely translated FLAG-Htt was immunoprecipitated and as such was associated with the $H t t$ mRNA solely through the whole translational apparatus. To rule out this possibility, we dissociated ribosomes prior to FLAG affinity purification by the addition of EDTA to total cytoplasmic lysates [17]. Addition of EDTA did not significantly affect the amount of FLAG-Htt recovered from $F 7 Q$ affinity purifications, but reduced F140Q/+ recovery by approximately 50\% (Fig. 1a, lanes 13-16). However, EDTA pre-treatment nearly 
a)

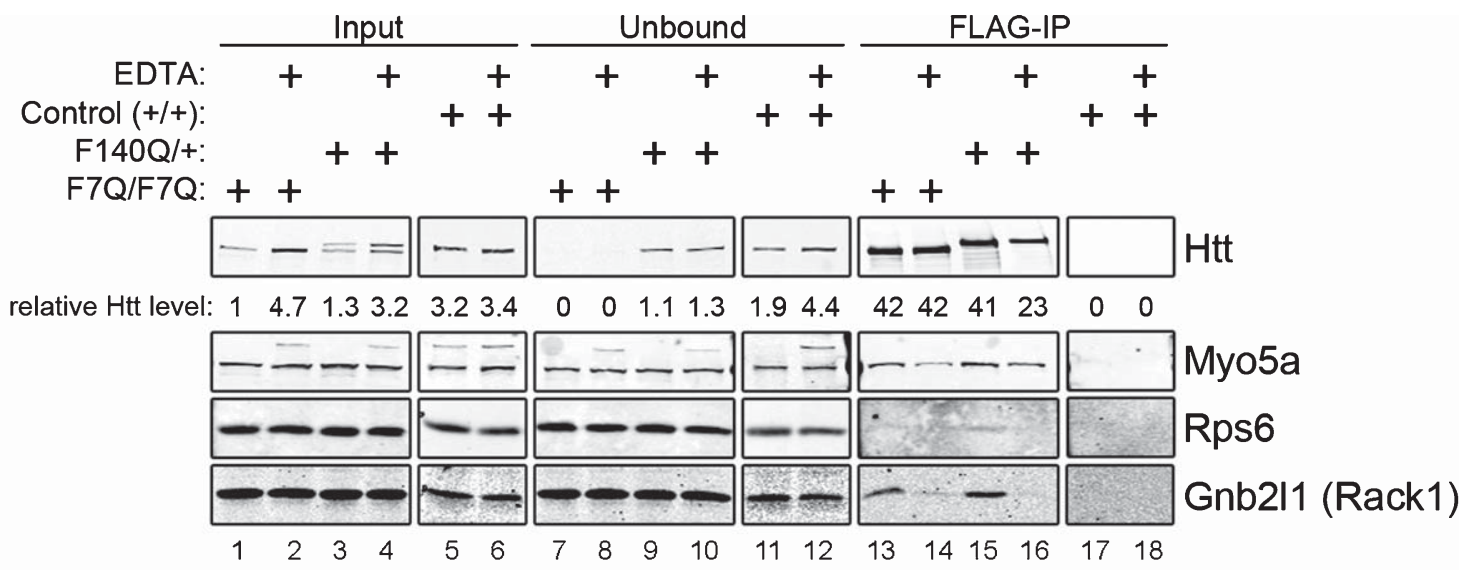

b)

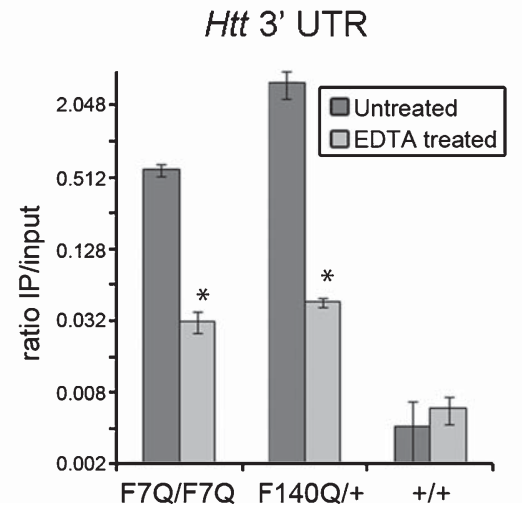

C)

\section{Htt 3' UTR}

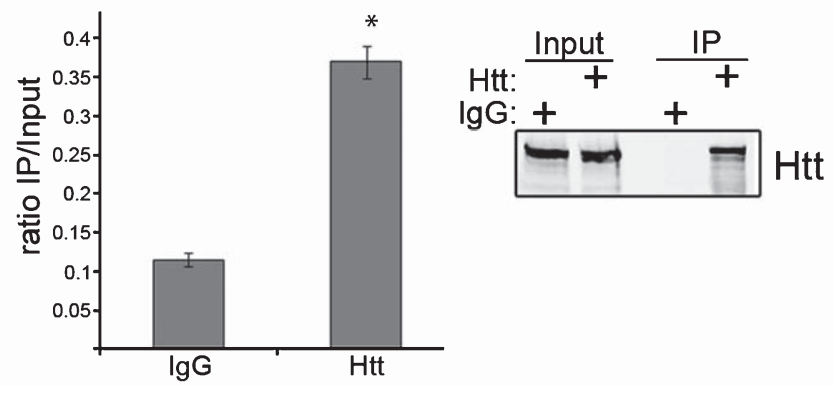

Fig. 1. Wild type and mutant Htt proteins associate with Htt mRNA. a) Htt association with ribosomal proteins depends on the presence of intact ribosomes. Western blots of input, unbound, and FLAG-immunoprecipitated proteins from negative control (Control), 3xFLAG7Q/3xFLAG7Q (F7Q/F7Q), and 3xFLAG140Q/+(F140Q/+) total cytoplasmic lysates with or without 20 mM EDTA pre-treatment. $20 \%$ of total immunoprecipitated protein was loaded for $\mathrm{Htt}$ blot and $80 \%$ for other blots. The numbers below indicate the amount of $\mathrm{Htt}$ in each lane relative to the amount of $\mathrm{Htt}$ present in $30 \mu \mathrm{g}$ F7Q/F7Q inputs. b) $\mathrm{Htt}$ mRNA is substantially enriched in FLAG-Htt affinity purifications. The graph shows quantification of RT-qPCR data for indicated samples relative to the amount of $H t t$ mRNA present in $1 \mu \mathrm{g}$ of total RNA from input samples. RT-qPCR was performed with primers specific to the $H t t 3^{\prime}$ UTR. ${ }^{*} P<0.05, t$-test c) Htt immunoprecipitation recovers more $H t t$ mRNA than IgG control immunoprecipitations. Western blot shows that Htt immunoprecipitation pulls out Htt from a Neuro2a cell lysate while the IgG negative control does not. The graph shows the relative enrichment of $H t t$ ' UTR in negative control (IgG) and $\mathrm{Htt}$ immunoprecipitation compared with $1 \mu \mathrm{g}$ of total RNA from the input. ${ }^{*} P<0.05$, $t$-test.

completely abolished the wild type and mutant $\mathrm{Htt}$ interactions with ribosome-associated proteins Rps6 and Gnb211 (Rack1)(Fig. 1a, lanes 13-16). In contrast, the Htt-Myo5a interaction was not completely abolished by this treatment. These data show that EDTA addition does not prevent FLAG-mediated purification of FLAG-Htt and that interactions of Htt with ribosomal proteins is largely dependent upon the integrity of the whole ribosome.

We next measured the levels of $H t t$ mRNA present in FLAG-Htt purifications by quantifying it relative to the amount of $H t t$ mRNA present in $1 \mu \mathrm{g}$ of total input RNA, which is expressed as a numerical ratio (Fig. 1b). FLAG IP of both wild type (F7Q/F7Q) and mutant (F140Q/+) Htt protein recovered significant amounts of $H t t$ mRNA compared with negative control. Although addition of EDTA drastically reduced the enrichment ratio of $\mathrm{Htt}$ mRNA for both wild type and mutant, these ratios were still 12 and 24 times greater than that of the negative control, for wild type and mutant, respectively (Fig. 1b, EDTA-treated). These data show that a pool of the Htt protein-Htt mRNA association occurs independently of ribosomal integrity. 
Furthermore, the enrichment of $H t t$ mRNA in EDTAtreated samples compared with the negative control argues against the possibility that the Htt protein$H t t$ mRNA association is an experimental artifact. To further substantiate the $\mathrm{Htt}$ protein- $\mathrm{Htt}$ mRNA interaction, we immunoprecipitated endogenous $\mathrm{Htt}$ from Neuro-2a cells and measured the amount of Htt 3' UTR present within control (IgG) and Htt immunoprecipitates (Htt) by RT-qPCR. Htt immunoprecipitates contained 3 times as much $\mathrm{Htt}$ 3' UTR as the negative control, indicating that Htt protein$H t t$ mRNA association does not occur through the FLAG tag.

\section{Htt protein co-localizes with Htt mRNA in neuronal cells}

We have previously shown that $\mathrm{Htt}$ co-localizes with the 3' UTR of $\beta$-actin and Bdnf mRNA in cultured rat neurons [8]. We hypothesized that the 3' UTR of $H t t$ is likely to contain element(s) responsible for associating with $\mathrm{Htt}$ protein. To confirm this possibility we examined the location of Htt with respect to a reporter construct harboring the short and long versions of the Htt 3' UTR in a mouse immortalized striatal precursor cell lines expressing wild-type $H t t\left(S T H d h^{7 Q / 7 Q}\right)$ or mutant $H t t\left(S T H d h^{111 Q / 111 Q}\right)$ [11]. Our assays employed the bipartite MS2 reporter system $[10,18]$ to visualize Htt protein with respect to a transfected mRNA containing the short and long versions of the Htt 3' UTR (Fig. 2a), preceded by the Renilla luciferase coding sequence and 24 tandem copies of the bacteriophage MS2 binding sequence. Control experiments indicated that Venus signal was primarily confined to the nucleus in wildtype and mutant $S T H d h$ cells transfected with the NLS-MS2-Venus reporter alone (Fig. 2b, Venus panels), indicating that the presence of the NLS on the Venus reporter confined Venus to the nucleus. In contrast, wild-type cells (Fig. 2b, $S T H d h^{7 Q / 7 Q}$ ) expressing both the NLS-MS2-Venus and an MS2 binding site-tagged $\mathrm{Htt}$ short 3' UTR displayed numerous large and small cytoplasmic puncta. Many of these cytoplasmic puncta co-localized with wildtype Htt (Fig. 2b, short UTR, arrowheads), evidenced by the appearance of yellow spots in the merged image. A recent cross-linking immunoprecipitation study of fragile-X mental retardation protein (Fmrp) identified $\mathrm{Htt}$ RNA as a target of Fmrp binding in mouse brains [17]. Consistent with this finding, we found co-localization of Fmrp with the Htt 3' UTR in this assay (Fig. 2b, short UTR, blue-green spots).
Furthermore, we observed several Htt short 3' UTR puncta, which were labeled for both Htt and Fmrp, suggesting that both proteins are capable of associating with the Htt short 3' UTR (Fig. 2b, upper panel, short UTR, arrow). Not surprisingly, wild-type cells expressing NLS-MS2-Venus and MS2 binding sitetagged $H t t$ long 3' UTR constructs also displayed numerous cytoplasmic puncta, which also stained positively for Htt and Fmrp (Fig. 2b, long UTR, arrow and arrowhead). These data demonstrate that the 3' UTR of Htt is sufficient to attract Htt protein to an exogenous mRNA in wild-type cells.

Mutant cells (Fig. 2b, STHdh ${ }^{111 Q / 111 Q}$ ) expressing NLS-MS2-Venus and MS2 binding site-tagged $H t t$ short 3' UTR also displayed cytoplasmic distribution of Venus signal, although the puncta were often larger and more concentrated than those observed in wild-type cells. Like wild-type cells, $\mathrm{Htt}$ short 3' UTR as reported by Venus signal was frequently found to co-localize with both Fmrp and Htt, particularly at the periphery of the cell. This peripheral localization of all three elements was also observed in mutant cells in which the localization of the long version of the Htt 3' UTR was monitored (Fig. 2b, long UTR). Finally, we transfected MS2 binding site-tagged $H t t$ long 3' UTR into rat cortical neurons and demonstrated co-localization with endogenous rat $\mathrm{Htt}$ by immunofluorescence (Fig. 2c).

\section{Htt represses gene expression through its 3' UTR sequence}

Next we sought to determine the effect that the 3' UTR of $\mathrm{Htt}$ might have on the expression of a linked GFP reporter. To do this, we generated GFP expression constructs containing either the SV40 polyadenylation signal alone, the short $H t t$ 3' UTR, or the long Htt 3' UTR (Fig. 3a). These constructs were transfected into Neuro-2a cells at equal molar ratios and GFP expression levels monitored by western blotting. Addition of the Htt short 3' UTR to the GFP expression construct led to a $57 \%$ reduction in GFP protein (Fig. 3b), while the long 3' UTR had an even larger inhibitory effect, reducing GFP expression by $95 \%$ compared with the empty control. These data agree with the results from [19] and support an inhibitory role for the 3' UTR of $\mathrm{Htt}$ on protein expression.

Given that Htt likely associates with its own mRNA and that much of this association is dependent upon the presence of intact ribosomes (Fig. 1a, b), we 
a)

NLS-MS2-Venus Venus

Htt short 3' UTR r. luciferase $0.5 \mathrm{~kb}$

Htt long 3' UTR

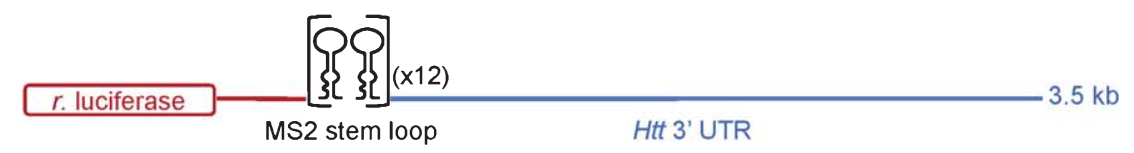

b)

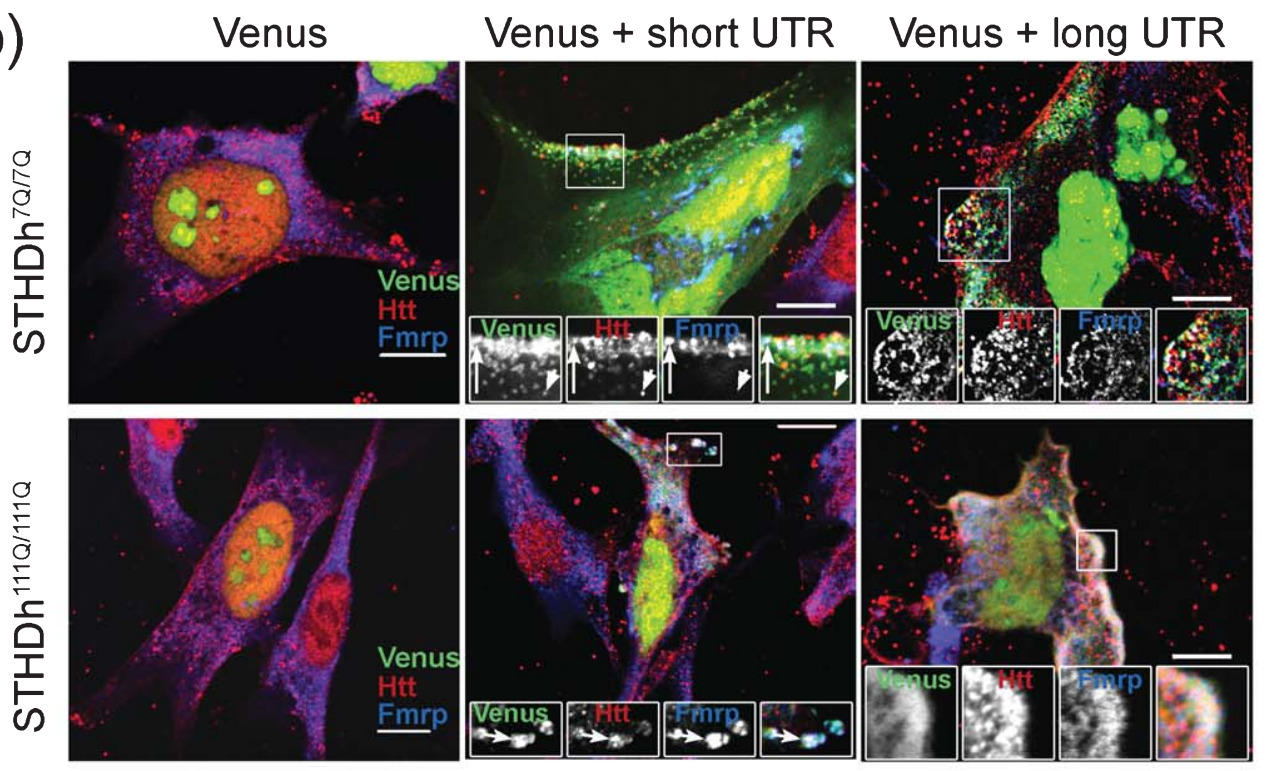

C) Venus + long UTR
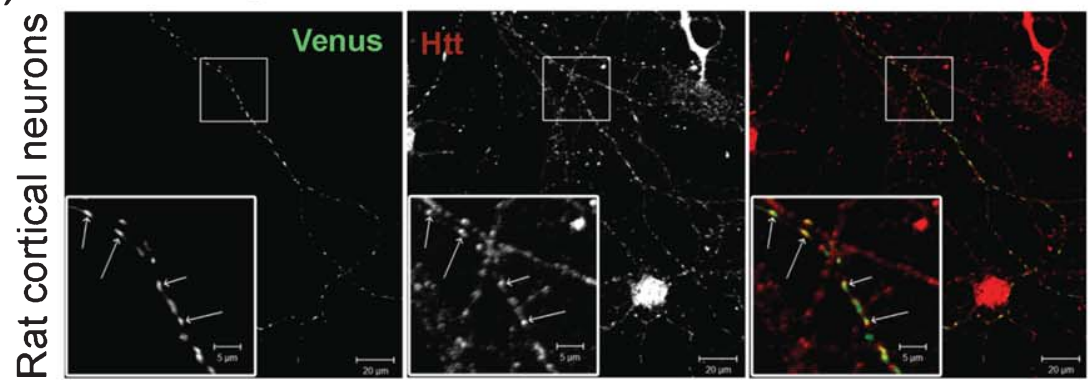

Fig. 2. MS2-tagged Htt 3' UTR co-localizes with Htt in wild type and mutant $H t t$ striatal precursor cell lines. a) Schematic shows the elements of the bipartite MS2 reporter system containing short or long Htt 3' UTR sequence. Control experiments express NLS-MS2-Venus alone. b) Htt and Fmrp co-localize with $H t t$ short and long reporters in wild-type $\left(S T H d h^{7 Q / 7 Q}\right)$ and mutant $\left(S T H d h^{111 Q / 111 Q}\right)$ striatal precursor cells. Merged images and individual channels for enlarged regions (boxes) are shown for each experimental combination. The arrows highlight instances of all three co-localization events, while the arrowhead shows Htt and Htt 3' UTR co-localization. Scale bars are $10 \mu \mathrm{m}$. c) Endogenous Htt co-localizes with long Htt 3' UTR in primary rat cortical neurons.

wanted to determine the effect that overexpression of Htt had on the levels of GFP expression in the context of the different Htt 3' UTRs. Overexpres- sion of full-length wild-type Htt (Htt17Q) led to a nearly $50 \%$ decrease in the levels of GFP-Htt short when compared with the control without exogenous 
a)

"empty"

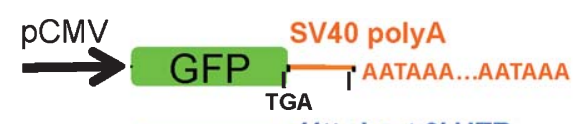

Htt "short"

$H$ tt "long"

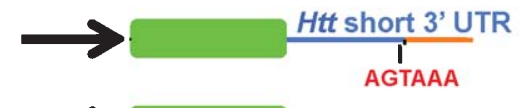

Htt long 3' UTR

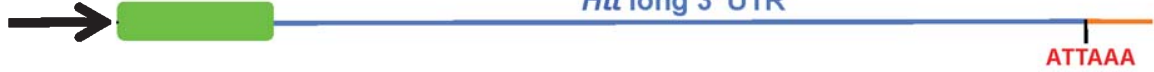

b)

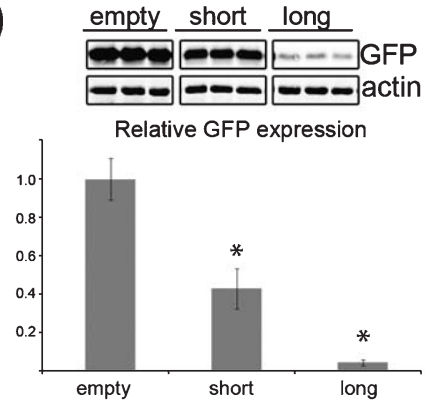

c)
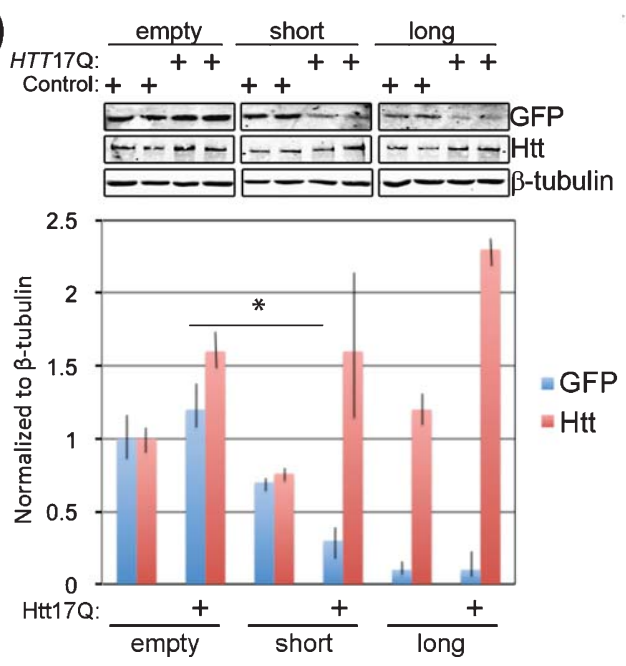
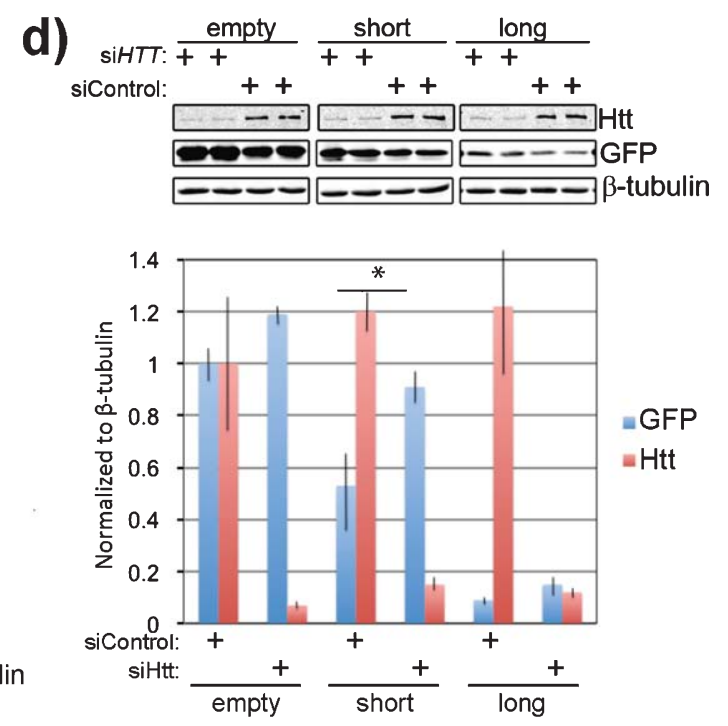

Fig. 3. The Htt 3' UTR reduces the expression of a GFP reporter in cis and Htt expression affects the levels of the GFP-Htt 3' UTR in trans. a) Schematic showing the design of GFP reporter constructs. Control (empty) GFP reporter contains the SV40 polyadenylation element alone that is present in pEGFP-C1. The "short" Htt 3' UTR GFP reporter contains the short version of the 3' UTR and similarly the "long" Htt 3' UTR GFP reporter contains the long version of the 3' UTR. Putative polyadenylation signals are labeled in red text and the engineered stop codon immediately after GFP coding sequence is also shown. b) Addition of the long Htt 3' UTR to the GFP reporter substantially reduces expression of GFP while the short version expresses at half the level of the control. Western blots show N2a cell experimental triplicates transfected with the indicated reporter constructs probed with antibodies to GFP and actin (loading control). The average intensity of GFP bands relative to the empty control are quantified in the bar graph and normalized to actin intensity. ${ }^{*} P<0.05$, $t$-test c) Overexpression of wild-type Htt $(H t t 17 Q)$ acts to further reduce the levels of the GFP reporter harboring the Htt 3' UTRs. Western blots show N2a cells with $(+H t t 17 Q)$ or without (Control) transfected wild-type Htt expression in the context of the expression of the indicated GFP reporter constructs. Blots were probed with antibodies to the indicated proteins and done in experimental triplicate. The graph shows the levels of GFP reporters relative to the sample transfected with vector alone (Control). ${ }^{*} P=0.05$, $t$-test d) Depletion of Htt with siRNA enhances GFP reporter harboring the Htt 3' UTR. Experiments were performed similar to c) except endogenous Htt was depleted by transfecting a mixture of siRNA to Htt. The graph shows the levels of GFP reporters relative to the sample transfected with a mixture of siRNA containing non-targeting sequences. ${ }^{*} P=0.1, t$-test. 
Htt expression (Fig. 3c). The already low levels of GFP-Htt long did not change with Htt overexpression.

To further test the possibility that $\mathrm{Htt}$ acts to negatively regulate the levels of protein produced from mRNA containing the Htt 3' UTR, we examined the effects of Htt knockdown on the expression of the GFP reporter constructs. siRNA-mediated knockdown of $\mathrm{Htt}$ led to a nearly 10 -fold decrease in the levels of Htt compared with the control knockdown (Fig. 3d, Htt level), and a 50\% increase in GFP-Htt short, and a $40 \%$ increase in GFP-Htt long expression levels compared with their levels in the control knockdown (Fig. 3d, GFP level). These results suggest that $\mathrm{Htt}$ has a role in the maintenance of its protein levels through the 3' UTR.

\section{RNA-Seq analysis of Htt co-purifying RNA identifies mis-spliced Htt exon $1 \mathrm{mRNA}$}

We performed RNA-Seq analysis of the same set of FLAG-immunoprecipitated samples from mouse brains previously subjected to microarray analysis. We indeed detected $\mathrm{Htt}$ mRNA sequences across the entire length of the gene at levels orders of magnitude greater than in the control immunoprecipitate (from normal mouse brains expressing no FLAGtagged proteins to control for non-specific binding to the FLAG antibody) in both wild type and mutant Htt immunoprecipitates (Fig. 4a). Furthermore, analysis of sequence reads in FLAG immunoprecipitates from $\mathrm{F} 140 \mathrm{Q} /+$ brains showed presence of nearly equal number of reads corresponding to endogenous wild-type mouse allele and FLAG-tagged knockin allele, which can be distinguished by a short human sequence present in the knock-in allele (data not shown). This provides further compelling evidence that $\mathrm{Htt}$ protein-Htt mRNA association does not occur through the FLAG tag or through pull down of nascent FLAG-Htt polypeptide. A recent report identified the presence of mis-spliced $\mathrm{Htt}$ mRNA containing the sequence of $\mathrm{Htt}$ exon 1 and beginning of intron 1 specifically in cells and tissues expressing mutant Htt protein [20]. This prompted us to look for mRNA corresponding to $\mathrm{Htt}$ intron 1 . We indeed detected sequence reads from intron 1 only in $\mathrm{Htt}$ immunoprecipitates obtained from 140Q-expressing mouse brains (Fig. 4b, c). These results indicate that Htt protein complex contains both full-length and mis-spliced $H t t$ mRNA.

Consistent with the above results, RNA-Seq analysis of FLAG-Htt140Q immunoprecipitates from mouse embryonic stem cells expressing the FLAG- tagged protein were also found to contain full-length and exon $1 \mathrm{Htt}$ mRNA (data not shown). Furthermore, immunoblotting of the FLAG-Htt immunoprecipitates demonstrated the presence of a polypeptide migrating at $\sim 80 \mathrm{kDa}$ (aberrant migration due to expanded polyQ sequence) detected by $\alpha$-FLAG and $\alpha$-polyQ antibodies (Fig. 5a, c), but not $\alpha$-Htt (2166) monoclonal antibody whose epitope is located Cterminal to the region encoded by exon 1 (Fig. 5b). This polypeptide was found only in cells expressing mutant $\mathrm{Htt}$, confirming the existence of the $\mathrm{N}$-terminal mutant Htt fragment encoded by the misspliced $H t t$ mRNA.

\section{DISCUSSION}

Here we have shown that Htt associates with its own mRNA, possibly through the 3' UTR. We do not yet know whether the interaction is direct or through other RNA binding proteins with which $\mathrm{Htt}$ co-purifies. This finding has interesting parallels to the discovery that the RNA binding protein TDP-43 regulates the levels of its own mRNA through the 3' UTR to keep them within a certain acceptable threshold [21], thereby preventing the toxic accumulation of the protein [22]. TDP-43 aggregation is found in amyotrophic lateral sclerosis (ALS) and Huntington's disease $[23,24]$. Mutations in TDP-43 are associated with both familial and sporadic ALS [25, 26]. The 3' UTR of TDP-43 is conspicuously long and conserved [21], and overexpression of wild-type TDP-43 is sufficient to kill cells [22]. Like TDP-43, mutant Htt causes tissue-specific cell death and dysfunction, mutant Htt forms intracellular aggregates [3], the 3' UTR of $H t t$ can be quite long in neurons [27, 28], and Htt seems to have some role in controlling the levels of its own mRNA.

The Htt 3' UTR contains many putative miRNA binding sites and as one would predict, the longer version contains more miRNA binding sites than the shorter version. Exogenous expression of miRNAs capable of binding to the Htt 3' UTR reduces the expression of a luciferase reporter fused to said UTR [19]. We found that a reporter mRNA harboring the $H t t$ 3' UTR, could localize to cytoplasmic concentrations of Ago2 (data not shown) and Fmrp, which likely represent stress granules or P-bodies [29]. Similarly, we found that the fusion of the Htt 3' UTR to a GFP reporter acted to substantially reduce the levels of GFP protein. Overexpression of wild-type Htt protein further decreased the GFP levels. We also found 
a)
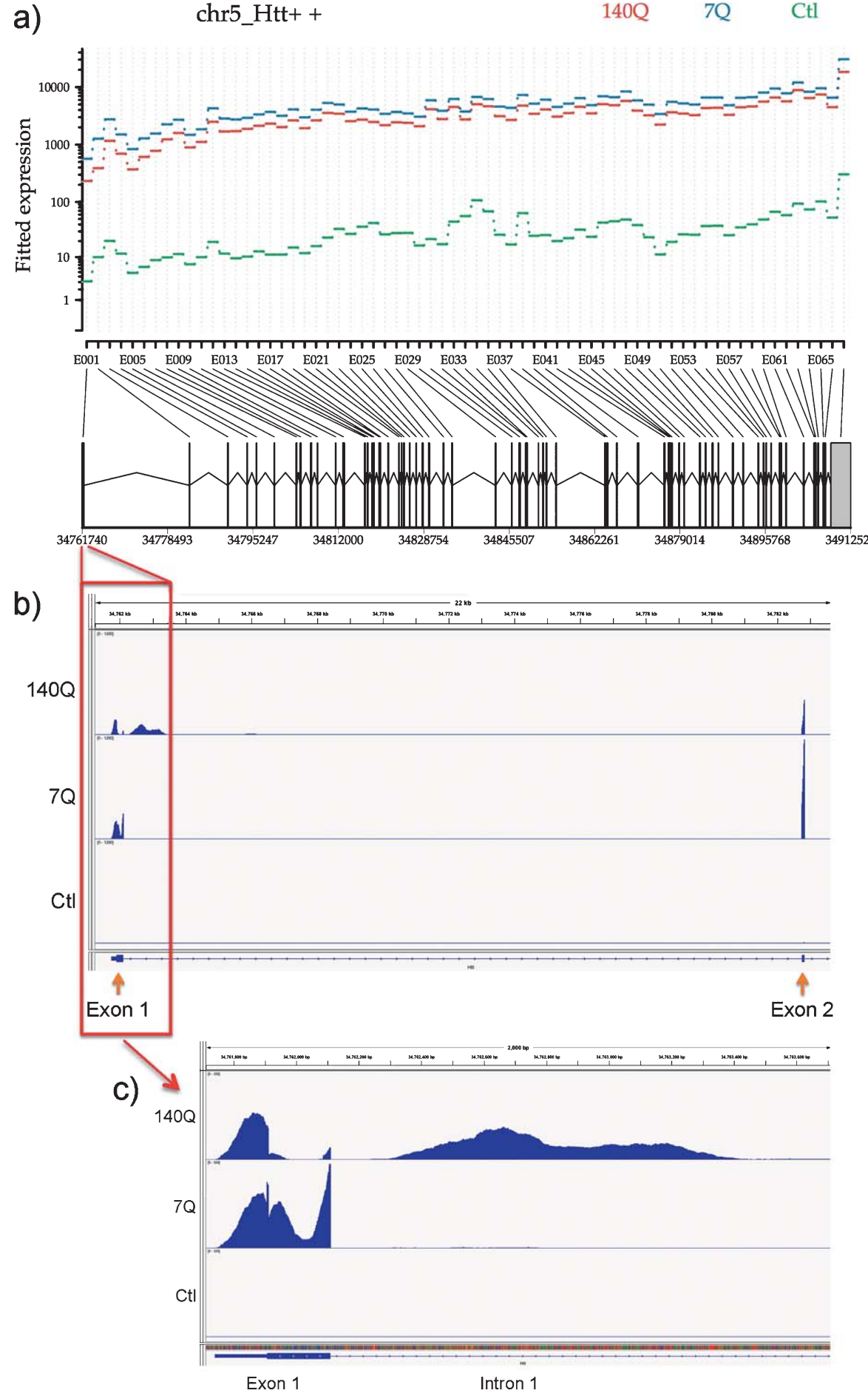

Fig. 4. RNA-Seq analysis of Htt immunoprecipitates reveals full-length Htt mRNA and mutant Htt-specific aberrantly spliced Htt mRNA associated with Htt protein. a) Differential expression of $H t t$ exons. The plot shows the fitted expression values of each of the exons of $H t t$ for each of the three conditions based on estimated dispersions as calculated by the statistical method DEXSeq. b) Read coverage for $H t t$ exon 1, intron 1, and exon 2. Coverage was normalized relative to the total number of mapped reads for each sample. Same scale was used for graphing all samples to illustrate relative expression levels. c) Read coverage for Htt exon 1, and the beginning of intron 1 . Coverage was normalized relative to the total number of mapped reads for each sample. Same scale was used for graphing all samples to illustrate relative expression levels. 

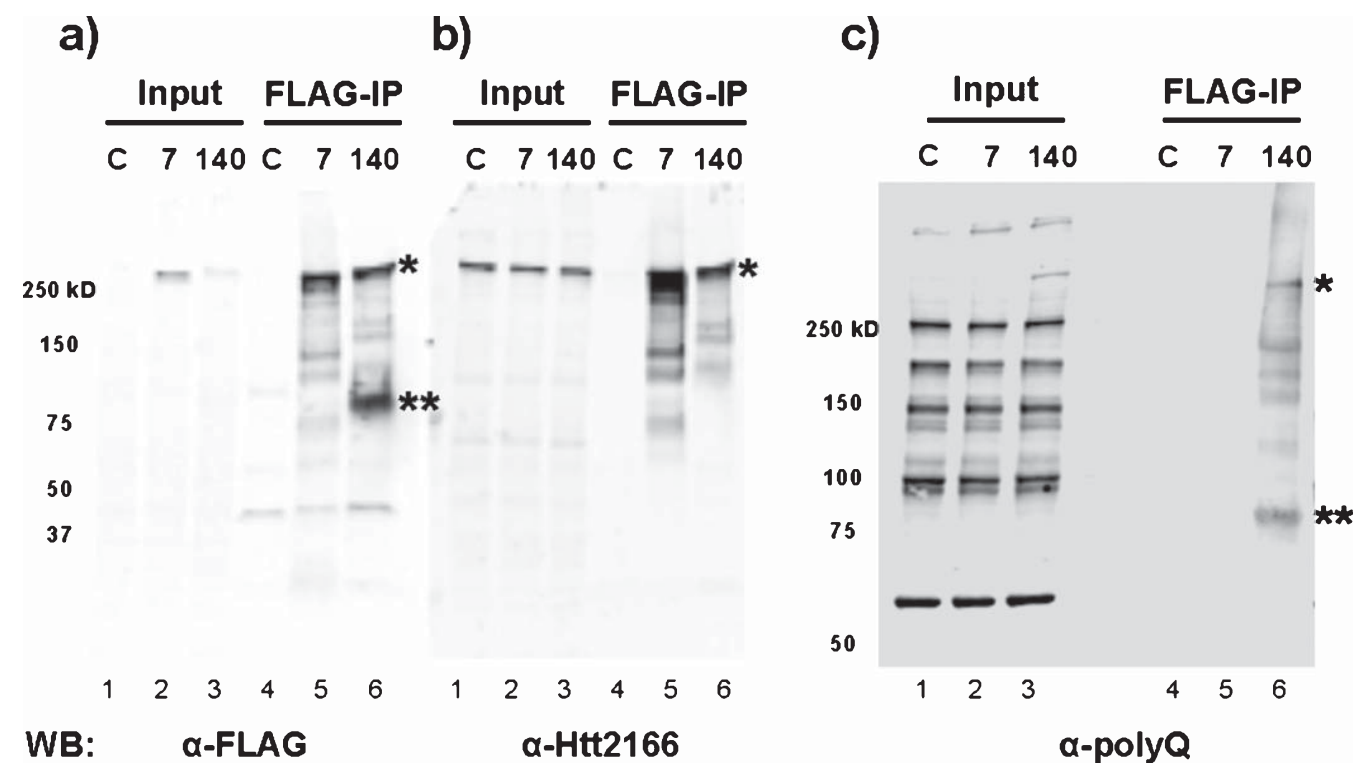

Fig. 5. Pathogenic exon $1 \mathrm{Htt}$ protein is only produced from mouse embryonic stem cells with expanded poly-glutamine tract. FLAG-IP was performed using total cell lysate extracted from wild-type (C), 3xFLAG-Htt ${ }^{7 \mathrm{Q} / 7 \mathrm{Q}}(7)$, and $3 x F L A G-H t^{140 Q / 7 Q}(140)$ mouse embryonic stem cells. Immunoblotting was performed with a) FLAG, b) Htt2166, and c) polyQ (3B5H10 clone) antibodies. The full-length 3xFLAG-tagged mutant Htt 140Q protein is detected with all three antibodies $\left(^{*}\right)$. The mis-spliced Htt exon 1 protein $\left(^{* *}\right)$ is detected by anti-FLAG and anti-polyQ antibodies, but not with anti-Htt2166 whose epitope falls outside of exon 1 . The mobility of this protein is retarded by the polyQ tract; it does not migrate to the predicted molecular weight. The western blots in panels a and b are from one gel run with duplicate samples, and the western blot in panel $\mathrm{c}$ is from a separate gel loaded with the same sample set. Both are 4-15\% polyacrylamide gradient gels.

that reduced Htt protein levels (by siRNA) acted to enhance the protein levels of a GFP reporter fused to the Htt 3' UTR. It is possible that Htt negatively influences the translation of mRNAs containing the Htt 3' UTR. However, given the complexity of biological processes, it is most likely that like TDP-43, multiple mechanisms exist to keep Htt expression at an appropriate level.

It was recently reported that a mis-spliced mRNA encoding the $\mathrm{N}$-terminal $\mathrm{Htt}$ exon 1 fragment is present specifically in cells and tissues expressing mutant Htt protein [20]. This prompted us to search for mRNAs mapping to introns of the $\mathrm{Htt}$ gene in our immunoprecipitates containing $\mathrm{Htt}$ in addition to full-length Htt mRNA that we first identified. Indeed, we detected both the mis-spliced mRNA containing the exon 1 sequence and its encoded polypeptide only in the immunoprecipitates containing the mutant $\mathrm{Htt}$ protein, confirming the above report. This finding raises the question of how $\mathrm{Htt}$ complex associates with both mis-spliced and fulllength $H t t$ mRNA since the two messages differ in their 3' UTR sequence. A motif search program (MEME) identified several short sequences in the 3' UTR from both mRNAs that included a putative bind- ing site for CELF4, a neuronal RNA binding protein reported to bind to $15 \%-20 \%$ of the brain transcriptome [30]. CELF4 binds specifically in the 3' UTR of target mRNAs enriched for synaptic functions. Like Htt, CELF4 is found in RNA granules suggesting a potential link to the Htt complex. Since the full-length and mis-spliced mRNAs have a common 5' UTR it is possible that $\mathrm{Htt}$ protein associates with both mRNAs through this region. We observed co-purification of wild type $\mathrm{Htt}$ protein with the full-length wild type $\mathrm{Htt}$ mRNA. We also found mutant Htt protein to co-purify with the full-length and mis-spliced $H t t$ mRNA. The two mRNAs might be differentially regulated by wild type or mutant Htt protein leading to potential changes in their levels thus contributing to HD pathogenesis.

The new discovery has raised a potentially significant role of Htt exon 1 in the pathogenesis of HD. Transgenic mice expressing Htt exon 1 with expanded polyQ sequence were made decades ago and have served as important mouse models of HD [31]. These animals show rapid progression to neurodegeneration and die within 3 months suggesting that mutant Htt exon 1 encoded polypeptide is highly toxic. By contrast, knock-in animals or BAC transgenic ani- 
mals expressing full-length mutant $\mathrm{Htt}$ show slower progression to disease and live nearly 2 years [32]. The exon $1 \mathrm{Htt}$ model has been thought of as an acute case of HD; however, given the new finding, toxicity associated with the $\mathrm{N}$-terminal polypeptide albeit at low levels could contribute to the disease progression in HD. Potential role for mutant exon 1 mRNA associated with the Htt complex remains to be investigated.

\section{ACKNOWLEDGMENTS}

This work was supported by National Institutes of Health Grant R01 NS061917 (to N.T.) and by a grant from the CHDI Foundation, Inc. (to N.T.). M.S.Y. was a recipient of Jan Vilcek Endowment Fellowship.

\section{CONFLICT OF INTEREST}

The authors have no conflict of interest to report.

\section{SUPPLEMENTARY MATERIAL}

The supplementary table and figure are available in the electronic version of this article: http://dx.doi.org/ 10.3233/JHD-150177.

\section{REFERENCES}

[1] A novel gene containing a trinucleotide repeat that is expanded and unstable on Huntington's disease chromosomes. The Huntington's Disease Collaborative Research Group. Cell. 1993;72(6):971-83.

[2] Andrew SE, Goldberg YP, Kremer B, Telenius H, Theilmann J, Adam S, et al. The relationship between trinucleotide (CAG) repeat length and clinical features of Huntington's disease. Nat Genet. 1993;4(4):398-403.

[3] Roos RA. Huntington's disease: A clinical review. Orphanet J Rare Dis. 2010;5(1):40.

[4] Culver BP, Savas JN, Park SK, Choi JH, Zheng S, Zeitlin SO, et al. Proteomic analysis of wild-type and mutant huntingtin-associated proteins in mouse brains identifies unique interactions and involvement in protein synthesis. J Biol Chem. 2012;287(26):21599-614.

[5] Imarisio S, Carmichael J, Korolchuk V, Chen CW, Saiki $\mathrm{S}$, Rose C, et al. Huntington's disease: From pathology and genetics to potential therapies. Biochem J. 2008; 412(2):191-209.

[6] Savas JN, Ma B, Deinhardt K, Culver BP, Restituito S, Wu $\mathrm{L}$, et al. A role for huntington disease protein in dendritic RNA granules. J Biol Chem. 2010;285(17):13142-53.

[7] Savas JN, Makusky A, Ottosen S, Baillat D, Then F, Krainc $\mathrm{D}$, et al. Huntington's disease protein contributes to RNA mediated gene silencing through association with Argonaute and P bodies. Proc Natl Acad Sci U S A. 2008; 105(31):10820-5.
[8] Ma B, Savas JN, Yu MS, Culver BP, Chao MV, Tanese N. Huntingtin mediates dendritic transport of beta-actin mRNA in rat neurons. Sci Rep. 2011;1:140.

[9] Ma B, Culver BP, Baj G, Tongiorgi E, Chao MV, Tanese N. Localization of BDNF mRNA with the Huntington's disease protein in rat brain. Mol Neurodegener. 2010;5:22.

[10] Bannai H, Fukatsu K, Mizutani A, Natsume T, Iemura S, Ikegami T, et al. An RNA-interacting protein, SYNCRIP (heterogeneous nuclear ribonuclear protein Q1/NSAP1) is a component of mRNA granule transported with inositol 1,4,5-trisphosphate receptor type $1 \mathrm{mRNA}$ in neuronal dendrites. J Biol Chem. 2004;279(51):53427-34.

[11] Trettel F, Rigamonti D, Hilditch-Maguire P, Wheeler VC, Sharp AH, Persichetti F, et al. Dominant phenotypes produced by the HD mutation in STHdh(Q111) striatal cells. Hum Mol Genet. 2000;9(19):2799-809.

[12] Ma B, Tanese N. Combined FISH and immunofluorescent staining methods to co-localize proteins and mRNA in neurons and brain tissue. Methods Mol Biol. 2013;1010:123-38.

[13] Dobin A, Davis CA, Schlesinger F, Drenkow J, Zaleski C, Jha S, et al. STAR: Ultrafast universal RNA-seq aligner. Bioinformatics. 2013;29(1):15-21.

[14] Anders S, Pyl PT, Huber W. HTSeq-a Python framework to work with high-throughput sequencing data. Bioinformatics. 2015;31(2):166-9.

[15] Anders S, Reyes A, Huber W. Detecting differential usage of exons from RNA-seq data. Genome Res. 2012;22(10): 2008-17.

[16] Zheng S, Ghitani N, Blackburn JS, Liu JP, Zeitlin SO. A series of N-terminal epitope tagged Hdh knock-in alleles expressing normal and mutant huntingtin: Their application to understanding the effect of increasing the length of normal Huntingtin's polyglutamine stretch on CAG140 mouse model pathogenesis. Mol Brain. 2012;5:28.

[17] Darnell JC, Van Driesche SJ, Zhang C, Hung KY, Mele A, Fraser CE, et al. FMRP stalls ribosomal translocation on mRNAs linked to synaptic function and autism. Cell. 2011;146(2):247-61.

[18] Bertrand E, Chartrand P, Schaefer M, Shenoy SM, Singer RH, Long RM. Localization of ASH1 mRNA particles in living yeast. Mol Cell. 1998;2(4):437-45.

[19] Sinha M, Ghose J, Bhattarcharyya NP. Micro RNA -214, 150,-146a and-125b target Huntingtin gene. RNA Biol. 2011;8(6):1005-21.

[20] Sathasivam K, Neueder A, Gipson TA, Landles C, Benjamin AC, Bondulich MK, et al. Aberrant splicing of HTT generates the pathogenic exon 1 protein in Huntington disease. Proc Natl Acad Sci U S A. 2013;110(6):2366-70.

[21] Ayala YM, De Conti L, Avendano-Vazquez SE, Dhir A, Romano M, D'Ambrogio A, et al. TDP-43 regulates its mRNA levels through a negative feedback loop. EMBO J. 2011;30(2):277-88.

[22] Wils H, Kleinberger G, Janssens J, Pereson S, Joris G, Cuijt I, et al. TDP-43 transgenic mice develop spastic paralysis and neuronal inclusions characteristic of ALS and frontotemporal lobar degeneration. Proc Natl Acad Sci U S A. 2010;107(8):3858-63.

[23] Neumann M, Sampathu DM, Kwong LK, Truax AC, Micsenyi MC, Chou TT, et al. Ubiquitinated TDP-43 in frontotemporal lobar degeneration and amyotrophic lateral sclerosis. Science. 2006;314(5796):130-3.

[24] Schwab C, Arai T, Hasegawa M, Yu S, McGeer PL. Colocalization of transactivation-responsive DNA-binding protein 43 and huntingtin in inclusions of Huntington disease. J Neuropathol Exp Neurol. 2008;67(12):1159-65. 
[25] Rutherford NJ, Zhang YJ, Baker M, Gass JM, Finch NA, $\mathrm{Xu}$ YF, et al. Novel mutations in TARDBP (TDP-43) in patients with familial amyotrophic lateral sclerosis. PLoS Genet. 2008;4(9):e1000193.

[26] Sreedharan J, Blair IP, Tripathi VB, Hu X, Vance C, Rogelj B, et al. TDP-43 mutations in familial and sporadic amyotrophic lateral sclerosis. Science. 2008;319(5870): 1668-72.

[27] Casanova E, Alonso-Llamazares A, Zamanillo D, Garate C, Calvo P, Chinchetru MA. Identification of a long huntingtin mRNA transcript in mouse brain. Brain Res. 1996;743(12):320-3.

[28] Li SH, Schilling G, Young WS, 3rd, Li XJ, Margolis RL, Stine OC, et al. Huntington's disease gene (IT15) is widely expressed in human and rat tissues. Neuron. 1993;11(5): 985-93.
[29] Buchan JR, Parker R. Eukaryotic stress granules: The ins and outs of translation. Mol Cell. 2009;36(6):932-41.

[30] Wagnon JL, Briese M, Sun W, Mahaffey CL, Curk T, Rot $\mathrm{G}$, et al. CELF4 regulates translation and local abundance of a vast set of mRNAs, including genes associated with regulation of synaptic function. PLoS Genet. 2012;8(11): e1003067.

[31] Mangiarini L, Sathasivam K, Seller M, Cozens B, Harper A, Hetherington C, et al. Exon 1 of the HD gene with an expanded CAG repeat is sufficient to cause a progressive neurological phenotype in transgenic mice. Cell. 1996; 87(3):493-506.

[32] Heng MY, Detloff PJ, Albin RL. Rodent genetic models of Huntington disease. Neurobiol Dis. 2008;32(1):1-9. 